\title{
Impact of Alternative Thermal and Non-Thermal Processing on the Enzymatic Activity of Papaya and Strawberry Nectars and Their Blends
}

\author{
Jeffrey G. Swada ${ }^{1 *}$, Jose I. Reyes De Corcuera ${ }^{2}$, Nicki J. Engeseth ${ }^{3}$ \\ 1. Department of Food Science and Human Nutrition, Michigan State University, 469 Wilson Rd., 106 Trout, \\ East Lansing, MI 48824, USA \\ 2. Department of Food Science and Technology, University of Georgia, 100 Cedar Street, No. 118 Athens, GA \\ 30602, USA \\ 3. Department of Food Science and Human Nutrition, University of Illinois at Urbana-Champaign, $905 \mathrm{~S}$. \\ Goodwin Avenue, 208 Bevier Urbana, IL 61801, USA \\ E-mail: swadajef@msu.edu, jireyes@uga.edu,engeseth@illinois.edu
}

Received: 17 December 2019; Accepted: 21 January 2020; Available online: 10 April 2020

\begin{abstract}
Pectin methylesterase (PME) in papaya nectar results in undesirable gel formation and peroxidase (POD) in strawberry nectar leads to nutrient loss, browning, and off-flavor production. Because of this, the effect of alternative processing techniques including ultra high temperature (UHT, $20-135^{\circ} \mathrm{C}, 1-3 \mathrm{~s}$ ), high pressure processing (HPP, 20 or $60^{\circ} \mathrm{C}, 200-600 \mathrm{MPa}$ ) and irradiation (0-10 kGy) on PME and POD activity in papaya and strawberry nectar and their respective blends were compared to traditional thermal processing $\left(80-130^{\circ} \mathrm{C}, 0-10\right.$ min). Traditional thermal $\left(110^{\circ} \mathrm{C}, 5 \mathrm{~min}, 71.5 \%\right.$ reduction) and $\mathrm{UHT}\left(110^{\circ} \mathrm{C}, 1-3 \mathrm{~s}, 98.0 \%\right.$ reduction) processing were able to sufficiently reduce PME activity and prevent gel formation in papaya nectar. PME reduction was enhanced by synergistic reductions in nectar blends after UHT at $80^{\circ} \mathrm{C}$. HPP was unable to prevent gel formation in papaya nectar, with enhanced activity at $400 \mathrm{MPa}$. Exposure of a blend 50P:50S to $10 \mathrm{kGy}$ irradiation prevented gel formation. UHT enhanced POD activity at $110^{\circ} \mathrm{C}$ and synergistic reductions resulted for POD activity in nectar blends after irradiation. These findings highlight the benefits of alternative processing in reducing enzyme activity in fruit nectars and nectar blends.
\end{abstract}

Keywords: Pectin methylesterase; Peroxidase; Papaya and strawberry; Ultra high temperature; High pressure processing; Irradiation.

\section{Introduction}

Papaya and strawberry nectars provide notably high nutritional value and antioxidant capacities that can enhance human health [1-3]. Although papaya and strawberries have great potential to enhance human health, they are seasonal fruits with short shelf lives. Over $50 \%$ of papaya does not reach consumers due to quality loss during harvesting and shipping; similarly, significant amounts of strawberries are lost due to softening and molding during shipping [4]. One main cause of this reduction in fruit quality is undesirable enzyme activity: both papaya and strawberry nectars naturally contain enzymes that can result in loss of consumer acceptance, lower nutrient retention, and overall decline in perceived quality if activities are not reduced through processing.

In papaya nectar, if PME activity is not sufficiently reduced, an undesirable gel forms and creates a product that is not well received by consumers [5, 6]. PME functions to de-esterify methoxy groups on pectin molecules within the cell wall, regulating properties of the cell wall and affecting the quality of the fruit [7]. This enzyme does not result in significant gel formation in strawberry nectar due to low concentrations of native PME, pectin, and calcium, as well as the low $\mathrm{pH}$ of the fruit [8-10]. In strawberry nectar, high POD activity is associated with nutrient loss, browning, off-flavor production, and reduced shelf life [11-13]. POD is an oxidoreductase which utilizes a variety of substrates and hydrogen donors (most commonly hydrogen peroxide) as part of their mechanism - specifically phenols in strawberry nectar [14-18].

Traditional thermal processing has proven effective at reducing these enzymatic activities. However, accompanying losses in nutrient content and product quality make this form of processing undesirable in these fruits [19-21]. Therefore, it is important to examine different alternative thermal and non-thermal processing techniques and their effect on PME and POD inactivation. UHT, HPP, and irradiation are alternative techniques that have been shown to reduce enzyme activity and as a result can provide additional strategies for processing papaya and strawberry nectars [22-24]. 
Though papaya and strawberry nectars exhibit high nutrient value and antioxidant capacity on their own, research suggests that a synergistic effect in terms of nutrient value may occur when fruits are blended together [25-32]. Blending fruit nectars may also enhance inactivation of enzymatic activity after processing. PME functions optimally at a $\mathrm{pH}$ of 6.9-7 [33], and when strawberry nectar ( $\mathrm{pH}$ 3-3.9) is blended with papaya nectar (pH 5.2-6), the resulting reduction in $\mathrm{pH}$ may inhibit the activity of PME compared to papaya nectar on its own $[21,34]$. Strawberry nectar contains high levels of phenolic compounds which act as the primary substrate for POD [14-18]. When strawberry nectar is blended with papaya nectar, the resulting dilution of POD’s substrate may also lead to reduction in enzyme activity [35].

The objective of this study was to evaluate how alternative thermal and non-thermal processing techniques such as UHT, HPP, and irradiation affect key deteriorative enzymes such as PME in papaya nectar and POD in strawberry nectar as compared to traditional thermal processing. In addition, possible synergistic reduction of enzymatic activity was explored through the blending of papaya and strawberry nectars.

\section{Materials and Methods}

\subsection{Materials}

$\mathrm{NaCl}$, Triton X-100, and hydrogen peroxide were acquired from Fisher Scientific (Fair Lawn, NJ). Highmethoxy pectin, $\mathrm{NaOH}$, Poly-vinyl-polypyrolidone (PVPP), p-phenylenediamine, and monobasic sodium phosphate were purchased from Sigma-Aldrich (St. Louis, MO). Dibasic sodium phosphate was obtained from J.T Baker Chemical Co. (Philipsburg, NJ). Polydimethylsiloxame 50 cSt was attained from Clearco Products (Bensalem, PA).

\subsection{Sample preparation and processing}

\subsubsection{Papaya and strawberry pulping}

Ripe papaya (Red Flesh, Product of Brazil, www.ugbp.com) and strawberry (Crimson Gold Strawberries, GPC - Grimes Produce Co., Plant City, FL) pieces were pulped using a Kitchenaid ${ }^{\mathrm{TM}}$ mixer (Whirlpool, St. Joseph, MI) equipped with a pulping attachment (Kitchenaid ${ }^{\mathrm{TM}}$ fruit and vegetable strainer). The pulp's initial total soluble solids concentration (SSC) was determined using a refractometer (Westover Scientific, Seattle, WA) and diluted as necessary to achieve $8^{\circ} \mathrm{Bx}$ and $6^{\circ} \mathrm{Bx}$ nectar for papaya and strawberry, respectively. The $\mathrm{pH}$ was also determined for the diluted nectar using an ion analyzer meter (Orion Research EA 920, Cambridge, MA). Diluted papaya nectar was immediately frozen at $-20^{\circ} \mathrm{C}$ until thermally or non-thermally processed and diluted strawberry nectar, made from frozen whole strawberries, was immediately thermally or non-thermally processed after pulping. After processing, samples were stored at $-20^{\circ} \mathrm{C}$ until analyzed.

\subsubsection{Control sample preparation}

All controls consisted of diluted papaya and strawberry nectar at $8^{\circ} \mathrm{Bx}$ and $6^{\circ} \mathrm{Bx}$, respectively, except UHT controls which consisted of diluted papaya and strawberry nectar at $2^{\circ} \mathrm{Bx}$ (Section 2.2.4). Controls traveled with treated samples, being handled and stored identically to treated samples, but not thermally or non-thermally processed.

\subsubsection{Traditional thermal processing}

Fruit nectar was placed into sealed capillary tubes (3 mm i.d.) and exposed to temperatures of 80, 90, 100, 110, 120 or $130^{\circ} \mathrm{C}$ for $0,0.5,1,2,3,5$, or $10 \mathrm{~min}$ (not all temperature/time combinations are presented) using a circulating silicone oil immersion bath. Polydimethylsiloxame was used as the immersion fluid due to its high boiling point and compatibility with the circulating immersion bath. Time began when the fruit nectar reached the target temperature; capillaries were immediately cooled in an ice bath after processing. The come up time was determined using an identical sealed capillary tube containing a wire thermocouple temperature probe (TipTemp, Burlington, NJ) placed equidistant from either end of the tube, which was then connected to an instant-read digital thermometer (Cole-Parmer Instrument Company, Vernon Hills, IL). Samples and thermocouple were simultaneously placed in the immersion bath and temperature was immediately recorded with processing time starting once the target processing temperature was reached. The temperature/time combinations were chosen to bracket traditional thermal processing conditions found in the literature [5,36].

\subsubsection{UHT processing}

Due to particle size restrictions imposed to ensure processing of samples, fruit pulping techniques were modified for this technique. Papaya and strawberry were pulped as outlined in Section 2.2.1. The fruit pulp was then additionally pureed in a Bella High Powered food processor (Sensio Inc., Canada) for $60 \mathrm{~s}$ to further reduce pulp particle size. The pureed nectar was adjusted to $2^{\circ} \mathrm{Bx}$ for both papaya and strawberry and either frozen at $-20^{\circ} \mathrm{C}$ 
until thawed or immediately UHT processed. Though samples were UHT processed at $2^{\circ} \mathrm{Bx}$, results are given as $8^{\circ} \mathrm{Bx}$ and $6^{\circ} \mathrm{Bx}$ for papaya and strawberry nectars, respectively.

Samples were exposed to 80,110 and $135^{\circ} \mathrm{C}$ for approximately $2 \mathrm{~s}$ (1-3 s due to machine limitations) using a pilot plant scale Armfield FT74T Miniature UHT Heat Exchanger (Armfield, Ringwood, UK). Specific temperature and time combinations were chosen to mirror parameters used to process similar fruits in the literature [23, 37-39], and to determine if this method of thermal processing was applicable to papaya and strawberry nectars.

\subsubsection{HPP}

The HPP system utilized consisted of a high pressure reactor, model U111, a high pressure micropump, model MP5, and a pump controller, MP5 micropump control unit (Unipress Equipment, Warsaw, Poland). Samples were run at $20^{\circ} \mathrm{C}$ or $60^{\circ} \mathrm{C}$ while applying 200, 400 or $600 \mathrm{MPa}$ pressure. An additional control sample was run simultaneously at atmospheric pressure at $20^{\circ} \mathrm{C}$ or $60^{\circ} \mathrm{C}$ to determine the true effects of added pressure to the processing of these samples [12, 13, 40].

Samples were thawed at $4^{\circ} \mathrm{C}$ four days prior to treatment with each sample being packed in a plastic pouch the day of processing. One sample was placed in a high pressure cell and pressurized and a control sample was placed in another identical high pressure cell that was not pressurized. Both cells were connected to the same water bath ensuring that the control and high pressure treated sample experienced identical temperature profiles. The samples were treated for 2 min with the following temperature and pressure profiles: processing began at $5^{\circ} \mathrm{C}$ under atmospheric pressure; samples were pressurized to set point (200, 400, or $600 \mathrm{MPa}$ ); temperature was raised to set point $\left(20\right.$ or $\left.60^{\circ} \mathrm{C}\right)$; once temperature reached $90 \%(\sim 40 \mathrm{~s})$ of the set temperature, incubation time began; samples were incubated $2 \mathrm{~min}$, and cooled to $15^{\circ} \mathrm{C}$ ( $\left.\sim 60 \mathrm{~s}\right)$ prior to depressurization, and finally samples were frozen immediately $\left(-20^{\circ} \mathrm{C}\right)$ after processing until analyzed.

\subsubsection{Irradiation}

Samples were exposed to 2.5, 5, 7.5, and $10 \mathrm{kGy}$ irradiation using a Gammacell 220 Cobalt-60 irradiator, model 375/2 (Ludlum Measurement, Inc., Sweetwater, TX) using a sealed radiation source, model GO 220E (CO-60, MDS Nordion, Inc., Ontario, CA). These exposure levels fall outside that of what is legally acceptable for processing of papaya and strawberry for consumer use at this current time [41]; however, a wide range of doses coupled with overall high doses were chosen specifically to determine the full impact that irradiation has on fruit. Parker et al. [6] conducted a similar experiment in which Rainbow ${ }^{\mathrm{TM}}$ and SunUp ${ }^{\mathrm{TM}}$ papaya were exposed to 5 and 7.5 kGy of irradiation.

Currently, irradiation doses of up to $1.0 \mathrm{kGy}$ are allowed for certain fruits. Typically papaya and melon are exposed to $0.25 \mathrm{kGy}$ of irradiation to eliminate the presence of fruit flies; these doses were not examined in this study since effects are widely understood in the literature [42]. Irradiation is also used for other foods such as white potatoes (0.05-0.15 kGy), fresh foods (1.0 kGy), uncooked beef/lamb/goat/pork (refrigerated: $4.5 \mathrm{kGy}$; frozen: 7.0 $\mathrm{kGy}$ ), spices (10 kGy), and dry/dehydrated aromatic vegetable substances (30.0 kGy) [41]. To achieve sterility it is estimated that up to $48 \mathrm{kGy}$ of irradiation is required [43]. Irradiation doses are regulated closely by the FDA due to the production of mutagenic or other potentially harmful compounds, which are not yet understood and may result from exposure to high levels of radiation [44]. Based on current FDA regulations on other food products and experiments already conducted, irradiation doses chosen here have potential for processing papaya and strawberry nectars.

\subsection{Sample characterization}

\subsubsection{Determination of PME activity}

This assay was adapted from Chang et al. [45] and measures the change in pH over time due to PME converting pectin to pectic acid. Fruit nectar was vortexed with $1.7 \mathrm{M} \mathrm{NaCl}(1: 4 \mathrm{w} / \mathrm{v})$. In a separate beaker, $1 \%$ pectin was adjusted to $\mathrm{pH} 7.5$ (with $0.02 \mathrm{M} \mathrm{NaOH}$ ). A water bath with heating coil housed the pectin solution, which was placed on a stir plate for complete mixing throughout the assay and temperature was maintained at $30^{\circ} \mathrm{C}$. The $\mathrm{NaCl}$ and fruit nectar mixture was added to the pectin solution creating a final $\mathrm{NaCl}$ concentration of $0.2 \mathrm{M}$, which is optimal for PME activity. The $\mathrm{pH}$ of the pectin solution was immediately adjusted back to 7.5 using small increments of $0.02 \mathrm{M} \mathrm{NaOH}$. Once the $\mathrm{pH}$ of the pectin solution was adjusted back to 7.5 this $\mathrm{pH}$ was maintained using $0.02 \mathrm{M} \mathrm{NaOH}$ over a span of $7 \mathrm{~min}$ with activity reported as $\mathrm{mEq} / \mathrm{min} / \mathrm{g}$ sample.

\subsubsection{Determination of POD activity}

POD activity was determined using protocols outlined by Cano and Hernandez [12] and Terefe et al. [13]. Fruit nectar was mixed with extraction solution (4\% PVPP, 1\% Titron X 100, in sodium phosphate buffer pH 6.5). The extract was homogenized at $4^{\circ} \mathrm{C}$ (ice bath) using a Polytron homogenizer (Model PT 10/35, Kinematica, Luzern, Switzerland). The extract was centrifuged at $1,250 \mathrm{x}$ g at $4^{\circ} \mathrm{C}$ for $90 \mathrm{~s}$. Supernatant was placed into Eppendorf 
tubes; if the extract was not clear, additional centrifugation was necessary. Sample extract was mixed with $0.05 \mathrm{M}$ sodium phosphate buffer ( $\mathrm{pH}$ 6.5). To this $1 \% \mathrm{p}$-phenylenediamine (in $0.05 \mathrm{M}$ sodium phosphate buffer) solution and $1.5 \%$ hydrogen peroxide was added. After vortexing, change in absorbance was monitored at $485 \mathrm{~nm}$ using a spectrophotometer (Spectronic E10185 Genesys 2, Spectronic Instruments, Garforth, UK). Optical Density (OD)/min/g of sample was recorded.

\subsection{Synergism experimental design and statistical analysis}

\subsubsection{Experimental design}

Three distinct blends were examined: 25\% papaya:75\% strawberry (25P:75S), 50\% papaya:50\% strawberry (50P:50S), and 75\% papaya:25\% strawberry (75P:25S). Each blend underwent thermal (UHT) and non-thermal (irradiation) processing as described in Section 2.2. After processing, enzymatic activity of the blends was analyzed as outlined in Section 2.3, and compared to 100\% papaya (100P) and 100\% strawberry (100S) controls.

\subsubsection{Simulation of expected values}

Samples were run in triplicate for each processing technique and respective treatment level. To increase the accuracy of simulated values a matrix model was applied to observed data [46]. This model generates every possible expected value based on the three replications collected for each treatment level. Each matrix generates nine expected values for a specific blend at a specific treatment level. These expected values were averaged and statistically analyzed to determine if they are significantly different from observed values.

\subsubsection{Statistical analysis}

Analysis of Variance (ANOVA) was used to determine significant differences at a $95 \%$ confidence interval ( $\alpha$ $\leq 0.05)$ using Statistical Analysis Software (SAS Institute, Inc., Cary, NC). Tukey's Studentized Range (HSD) and Scheffe's mean separation was also utilized where necessary using a 95\% confidence interval $(\alpha \leq 0.05)$. All basic statistical calculations such as averages, standard deviations, standard errors, and coefficients of variance were calculated using Microsoft Office Excel 2003

Once expected values were simulated, statistical analysis was required to determine if a difference existed between observed and expected values. In the first stage of analysis, all data were tested for normality. If the data were found to be normal ( $p>0.05$ ), the t-test procedure was utilized [46]. Since observed values and expected values came from different distributions, with potentially different sources of variability, equality of variance testing was performed to determine which type of t-test to use. To determine equality of variance, the Folded $\mathrm{F}$ value was calculated. If $(\mathrm{p}>\mathrm{F}$ ) was greater than 0.05 , variance of the two means was determined to not be significantly different, and a Pooled t-test was used. If ( $p>$ F) was less than 0.05 , variance between the two means was found to be unequal and the Satterthwaite t-test was used. In either case, if $(p>t)$ was greater than 0.05 , observed values were not significantly different from expected values, and if $(p>t)$ was less than 0.05 , observed values were found to be significantly different from expected values.

If sample data was not found to be normally distributed $(\mathrm{p}<0.05)$, then the Wilcoxon Rank Test (Mann-Whitney U-test) was utilized [46]. If $(p>U)$ was greater than 0.05 , observed values were not significantly different from expected values, but if ( $p>U$ ) was less than 0.05 , observed values were significantly different from expected values.

Using the results from the above tests, observed values found to be significantly greater than expected values suggested a possible antagonistic reduction. Observed values significantly indifferent from expected values suggested a possible additive reduction. Observed values significantly less than expected values suggest a possible synergistic reduction [46].

\section{Results and Discussion}

\subsection{Impact of processing on PME activity in papaya nectar}

After traditional thermal processing, significant reduction in PME activity occurred in papaya nectar across all temperatures starting at 2 minutes, but with different rates of inactivation depending on the specific temperature examined (Figure 1). As temperature increased, rate of PME inactivation increased, consistent with Fraeye et al. [43]. Similar PME inactivations were also seen in an orange juice-milk beverage as temperature increased [47; 48]. At $80^{\circ} \mathrm{C}$, papaya $\mathrm{PME}$ activity significantly decreased (19.8\%) at 2 minutes, remained relatively constant at 5 minutes (23.7\% reduction), and significantly decreased (46.5\%) again at 10 minutes (Figure 1 ). At $110^{\circ} \mathrm{C}$, papaya PME activity significantly decreased (29.0\%) at 2 minutes and continued to decrease at 5 minutes (71.5\%) and 10 minutes (81.2\%). At $130^{\circ} \mathrm{C}$, PME activity in papaya significantly decreased (87.2\%) at 2 minutes, and then continued to decrease at 5 minutes (99.0\%) and 10 minutes (99.1\%). Gel formation slowed after processing at $80^{\circ} \mathrm{C}$, with strong gels forming after 48 hours in the samples processed for 2 and 5 minutes (Table 1 ). In samples 
processed for 10 minutes a strong gel did not form until after 72 hours. Processing for 2 minutes at $110^{\circ} \mathrm{C}$ prevented gel formation up to 48 hours with slight gel formation after 72 hours, but at 5 and 10 minutes gel formation was prevented completely. All processing times resulted in complete prevention of gel formation at $130^{\circ} \mathrm{C}$. Based on these observations, in order to completely prevent undesirable gel formation in papaya nectar, a temperature-time combination of $110^{\circ} \mathrm{C}$ for 5 minutes is sufficient.

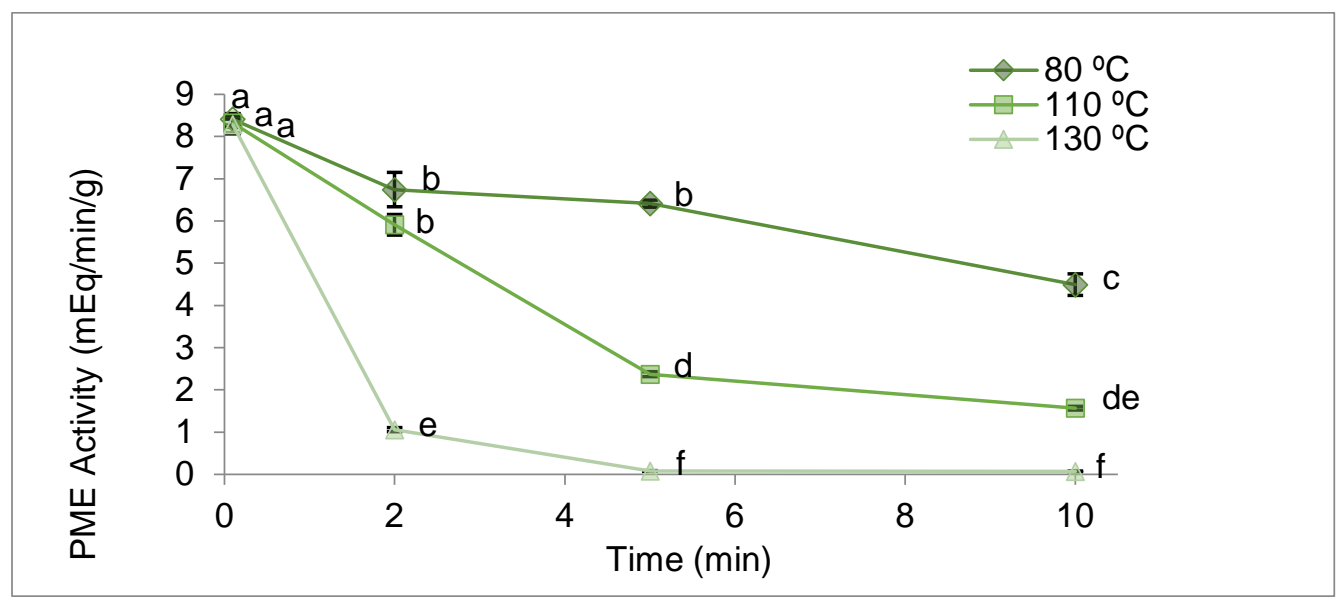

Figure 1. PME given as (mEq NaOH/min/g) for papaya nectar after thermal processing. Results given as (average \pm SE) x 1000. Values with the same letters are not significantly different (Tukey’s Studentized Range (HSD) and Scheffe's Test $(\alpha \leq 0.05))$.

Table 1. Gel formation for $100 \%$ papaya after traditional thermal processing

\begin{tabular}{|c|c|c|c|c|}
\hline & & 24 hours & 48 hours & 72 hours \\
\hline \multirow[t]{5}{*}{ Time (min) at $80^{\circ} \mathrm{C}$} & FF & SG & SG & SG \\
\hline & 0 & SG & SG & SG \\
\hline & 2 & LG & SG & SG \\
\hline & 5 & LG & SG & SG \\
\hline & 10 & LG & LG & SG \\
\hline \multirow[t]{4}{*}{ Time (min) at $110^{\circ} \mathrm{C}$} & 0 & SG & SG & SG \\
\hline & 2 & NG & NG & LG \\
\hline & 5 & NG & NG & NG \\
\hline & 10 & NG & NG & NG \\
\hline \multirow[t]{4}{*}{ Time (min) at $130{ }^{\circ} \mathrm{C}$} & 0 & SG & SG & SG \\
\hline & 2 & NG & NG & NG \\
\hline & 5 & NG & NG & NG \\
\hline & 10 & NG & NG & NG \\
\hline
\end{tabular}

* FF = Fresh fruit, $\mathrm{NG}=$ No gelling, $\mathrm{LG}=$ Slight gelling, $\mathrm{SG}=$ Strong gelling. All samples were compared to pectin solution controls.

Table 2. Gel formation for $100 \%$ papaya after high pressure processing (HPP, both pressure treated and atmospheric (atm) controls)

\begin{tabular}{cccc}
\hline Temperature $\left({ }^{\circ} \mathrm{C}\right)$, Pressure (MPa) & 24 hours & 48 hours & 72 hours \\
\hline FF & LG & LG & LG \\
20, atm & LG & LG & SG \\
20,400 & LG & SG & SG \\
20, atm & LG & LG & SG \\
20,600 & LG & LG & SG \\
20, atm & NG & LG & SG \\
60,200 & LG & LG & SG \\
60, atm & LG & LG & SG \\
60,400 & LG & SG & SG \\
60, atm & LG & LG & SG \\
60,600 & LG & LG & LG \\
60, atm & NG & LG & SG \\
\hline
\end{tabular}

* FF = Fresh fruit, NG = No gelling, LG = Slight gelling, $\mathrm{SG}=$ Strong gelling. All samples were compared to pectin solution controls. 
Table 3. Gel formation and $\mathrm{pH}$ for $100 \%$ papaya and strawberry nectar controls and their respective blends (25P:75S, 50P:50S, 75P:25S) after ultra high temperature (UHT) processing

\begin{tabular}{|c|c|c|c|c|c|c|c|c|c|c|c|c|c|c|c|c|c|c|c|}
\hline \multirow{2}{*}{$\begin{array}{c}\text { Temperat } \\
\text { ure }\left({ }^{\circ} \mathrm{C}\right)\end{array}$} & \multicolumn{3}{|c|}{$100 \mathrm{P}$} & \multicolumn{4}{|c|}{$100 \mathrm{~S}$} & \multicolumn{4}{|c|}{$25 \mathrm{P}: 75 \mathrm{~S}$} & \multicolumn{4}{|c|}{ 50P:50S } & \multicolumn{4}{|c|}{$75 P: 25 S$} \\
\hline & $24 \mathrm{~h} 48 \mathrm{~h}$ & $72 \mathrm{~h}$ & $\mathrm{pH}$ & $24 \mathrm{~h}$ & $48 \mathrm{~h}$ & $72 \mathrm{~h}$ & $\mathrm{H}$ & th & $48 \mathrm{~h}$ & $72 \mathrm{~h}$ & $\mathrm{H}$ & h & $48 \mathrm{~h}$ & $72 \mathrm{~h}$ & $\mathrm{pH}$ & $4 \mathrm{~h}$ & $48 \mathrm{~h}$ & $72 \mathrm{~h}$ & $\mathrm{H}$ \\
\hline $\mathrm{FF}$ & SG & SG & 5.31 & NG & NG & NG & 3.47 & & & & & & & & & & & & \\
\hline 20 & NG NG & LG & 5.3 & NG & NG & NG & 3.48 & NG & NG & NG & & & NG & LG & & NG & NG & LG & 4.7 \\
\hline 80 & NG NG & LG & 5.36 & NG & NG & NG & 3.57 & NG & NG & NG & 3.96 & NG & NG & NG & 4.19 & NG & NG & NG & 4.67 \\
\hline 110 & NG NG & NG & 5.52 & NG & NG & NG & 3.58 & NG & NG & NG & 4.02 & NG & NG & NG & 4.3 & NG & NG & NG & 4.7 \\
\hline 135 & NG NG & NG & 6.02 & NG & NG & NG & 3.67 & NG & NG & NG & 4.1 & NG & NG & NG & 4.4 & NG & NG & NG & 4.76 \\
\hline
\end{tabular}

* FF = Fresh fruit, $\mathrm{NG}=$ No gelling, $\mathrm{LG}=$ Slight gelling, $\mathrm{SG}=$ Strong gelling. All samples were compared to pectin solution controls.

Table 4. Gel formation and $\mathrm{pH}$ for $100 \%$ papaya and strawberry nectar controls and their respective blends (25P:75S, 50P:50S, 75P:25S) after irradiation processing

\begin{tabular}{|c|c|c|c|c|c|c|c|c|c|c|c|c|c|c|c|c|c|c|c|c|}
\hline \multirow{2}{*}{$\begin{array}{l}\text { Radiation } \\
\text { Dose } \\
\text { (kGy) }\end{array}$} & \multicolumn{4}{|c|}{$100 \mathrm{P}$} & \multicolumn{4}{|c|}{$100 \mathrm{~S}$} & \multicolumn{4}{|c|}{ 25P:75S } & \multicolumn{4}{|c|}{ 50P:50S } & \multicolumn{4}{|c|}{$75 \mathrm{P}: 25 \mathrm{~S}$} \\
\hline & $24 \mathrm{~h}$ & $48 \mathrm{~h}$ & $72 \mathrm{~h}$ & $\mathrm{pH}$ & $24 \mathrm{~h}$ & $48 \mathrm{~h}$ & $72 \mathrm{~h}$ & $\mathrm{pH}$ & $24 \mathrm{~h}$ & $48 \mathrm{~h}$ & $72 \mathrm{~h}$ & $\mathrm{pH}$ & $24 \mathrm{~h}$ & $48 \mathrm{~h}$ & $72 \mathrm{~h}$ & $\mathrm{pH}$ & $24 \mathrm{~h}$ & $48 \mathrm{~h}$ & $72 \mathrm{~h}$ & $\mathrm{pH}$ \\
\hline FF & SG & SG & SG & 5.31 & NG & NG & NG & 3.61 & & & & & & & & & & & & \\
\hline 0 & SG & SG & SG & 5.3 & NG & NG & NG & 3.62 & NG & NG & NG & 3.87 & LG & LG & LG & 4.11 & LG & SG & SG & 4.6 \\
\hline 2.5 & SG & SG & SG & 5.32 & NG & NG & NG & 3.63 & NG & NG & NG & 3.88 & LG & LG & LG & 4.12 & LG & LG & SG & 4.59 \\
\hline 5 & SG & SG & SG & 5.31 & NG & NG & NG & 3.62 & NG & NG & NG & 3.88 & LG & LG & LG & 4.11 & LG & LG & LG & 4.61 \\
\hline 7.5 & LG & LG & SG & 5.32 & NG & NG & NG & 3.62 & NG & NG & NG & 3.88 & LG & LG & LG & 4.11 & LG & LG & LG & 4.59 \\
\hline 10 & LG & LG & SG & 5.32 & NG & NG & NG & 3.63 & NG & NG & NG & 3.88 & NG & NG & NG & 4.11 & LG & LG & LG & 4.61 \\
\hline
\end{tabular}

$* \mathrm{FF}=$ Fresh fruit, $\mathrm{NG}=$ No gelling, $\mathrm{LG}=$ Slight gelling, $\mathrm{SG}=$ Strong gelling. All samples were compared to pectin solution controls.

UHT resulted in significant reduction in PME activity in papaya nectar (Table 5). As temperature increased from $20^{\circ} \mathrm{C}$ to $80^{\circ} \mathrm{C}$, there was a slight reduction in papaya $\mathrm{PME}$ activity $(21.2 \%)$, but at $110^{\circ} \mathrm{C}$ and $135^{\circ} \mathrm{C}$, significant reductions of $98.0 \%$ and $98.8 \%$ were observed, respectively. Gel formation in papaya nectar was completely prevented after UHT at $110^{\circ} \mathrm{C}$ (Table 1-4), consistent with Sanchez-Vega et al. [23].

Table 5. Observed vs. expected PME given as ( $\mathrm{mEq} \mathrm{NaOH} / \mathrm{min} / \mathrm{g}$ ) for $100 \%$ papaya and strawberry nectar controls and their respective blends (25P:75S, 50P:50S, 75P:25S) after ultra high temperature (UHT) and irradiation processing.

\begin{tabular}{|c|c|c|c|c|c|c|c|c|}
\hline & \multirow[t]{2}{*}{$100 \mathrm{P}$} & \multirow[t]{2}{*}{$100 \mathrm{~S}$} & \multicolumn{2}{|c|}{ 25P:75S } & \multicolumn{2}{|c|}{ 50P:50S } & \multicolumn{2}{|c|}{$75 \mathrm{P}: 25 \mathrm{~S}$} \\
\hline & & & Observed & Expected & Observed & Expected & Observed & Expected \\
\hline \multicolumn{9}{|c|}{ Temperature $\left({ }^{\circ} \mathrm{C}\right)$} \\
\hline 20 & $\begin{array}{c}9.60^{a} \pm \\
0.221\end{array}$ & $\begin{array}{c}1.24^{\mathrm{a}} \pm \\
0.703\end{array}$ & $\begin{array}{c}3.25^{a} \pm \\
0.190\end{array}$ & $\begin{array}{c}3.33 \pm \\
0.327\end{array}$ & $\begin{array}{c}5.36^{a} \pm \\
0.187\end{array}$ & $\begin{array}{l}5.42 \pm \\
0.0564\end{array}$ & $\begin{array}{l}7.41^{a} \pm \\
0.0723\end{array}$ & $\begin{array}{l}7.51 \pm \\
0.083\end{array}$ \\
\hline 80 & $\begin{array}{c}9.34^{a} \pm \\
0.254\end{array}$ & $\begin{array}{l}0.703^{b} \pm \\
9.8 \times 10^{-3}\end{array}$ & $\begin{array}{c}1.79^{b} \pm \\
0.684\end{array}$ & $\begin{array}{l}2.87 * \pm \\
0.32\end{array}$ & $\begin{array}{l}2.95^{b} \pm \\
0.0909\end{array}$ & $\begin{array}{l}5.05 * \pm \\
0.0635\end{array}$ & $\begin{array}{c}0.464^{b} \pm \\
7.73 \times 10^{-3}\end{array}$ & $\begin{array}{l}7.22 * \pm \\
0.0952\end{array}$ \\
\hline 110 & $\begin{array}{c}0.194^{b} \pm \\
3.17 \times 10^{-3}\end{array}$ & $\begin{array}{c}0.143^{\mathrm{c}} \pm \\
9.55 \times 10^{-5}\end{array}$ & $\begin{array}{c}0.207^{c} \pm \\
6.92 \times 10^{-5}\end{array}$ & $\begin{array}{l}0.155^{*} \pm \\
3.97 \times 10^{-4}\end{array}$ & $\begin{array}{c}0.214^{c} \pm \\
3.21 \times 10^{-5}\end{array}$ & $\begin{array}{c}0.168 * \pm \\
7.92 \times 10^{-4}\end{array}$ & $\begin{array}{c}0.228^{c} \\
\pm 8.55 \times 10^{-5}\end{array}$ & $\begin{array}{l}0.181^{*} \pm \\
1.19 \times 10^{-3}\end{array}$ \\
\hline 135 & $\begin{array}{c}0.114^{b} \pm \\
1.36 \times 10^{-15}\end{array}$ & $\begin{array}{l}0.0876^{\mathrm{c}} \pm \\
1.90 \times 10^{-3}\end{array}$ & $\begin{array}{c}0.207^{c} \pm \\
1.49 \times 10^{-4}\end{array}$ & $\begin{array}{l}0.0943^{*} \pm \\
7.14 \times 10^{-4}\end{array}$ & $\begin{array}{c}0.214^{c} \pm \\
1.50 \times 10^{-4}\end{array}$ & $\begin{array}{c}0.101^{*} \pm \\
4.76 \times 10^{-4}\end{array}$ & $\begin{array}{c}0.186^{c} \pm \\
3.25 \times 10^{-3}\end{array}$ & $\begin{array}{c}0.108^{*} \pm \\
2.38 \times 10^{-4}\end{array}$ \\
\hline \multicolumn{9}{|c|}{$\begin{array}{l}\text { Radiation Dose } \\
\text { (kGy) }\end{array}$} \\
\hline 0 & $\begin{array}{c}8.89^{a} \pm \\
0.366\end{array}$ & $\begin{array}{c}0.713^{\mathrm{a}} \pm \\
3.79 \times 10^{-4}\end{array}$ & $\begin{array}{l}2.76^{a} \pm \\
0.0486\end{array}$ & $\begin{array}{l}2.76 \pm \\
0.0458\end{array}$ & $\begin{array}{c}4.27^{a} \pm \\
3.13 \times 10^{-3}\end{array}$ & $\begin{array}{l}4.80 * \pm \\
0.0916\end{array}$ & $\begin{array}{l}5.04^{a} \pm \\
0.0486\end{array}$ & $\begin{array}{c}6.85^{*} \pm \\
0.137\end{array}$ \\
\hline 2.5 & $\begin{array}{c}6.37^{b} \pm \\
0.212\end{array}$ & $\begin{array}{c}0.677^{\mathrm{ab}} \pm \\
0.0201\end{array}$ & $\begin{array}{c}2.69^{a} \pm \\
0.120\end{array}$ & $\begin{array}{l}2.1^{*} \pm \\
0.0276\end{array}$ & $\begin{array}{c}4.13^{b} \pm \\
2.17 \times 10^{-3}\end{array}$ & $\begin{array}{l}3.52 * \pm \\
0.0534\end{array}$ & $\begin{array}{l}4.87^{a} \pm \\
0.0254\end{array}$ & $\begin{array}{l}4.95 \pm \\
0.0797\end{array}$ \\
\hline 5 & $\begin{array}{c}6.05^{b} \pm \\
0.188\end{array}$ & $\begin{array}{c}0.643^{\mathrm{b}} \pm \\
2.59 \times 10^{-3}\end{array}$ & $\begin{array}{l}2.49^{a b} \pm \\
0.045\end{array}$ & $\begin{array}{l}1.99 * \pm \\
0.0235\end{array}$ & $\begin{array}{c}3.99^{c} \\
\pm 4.02 \times 10^{-3}\end{array}$ & $\begin{array}{c}3.35 * \pm \\
0.047\end{array}$ & $\begin{array}{c}4.73^{a b} \\
\pm 0.0269\end{array}$ & $\begin{array}{c}4.7 \pm \\
0.0705\end{array}$ \\
\hline 7.5 & $\begin{array}{c}5.79^{b} \pm \\
0.00753\end{array}$ & $\begin{array}{c}0.628^{\mathrm{b}} \pm \\
0.0144\end{array}$ & $\begin{array}{c}2.23^{b c} \pm \\
0.0465\end{array}$ & $\begin{array}{l}1.92 * \pm \\
0.0109\end{array}$ & $\begin{array}{l}3.89^{d} \pm \\
0.0228\end{array}$ & $\begin{array}{l}3.21 * \pm \\
0.0192\end{array}$ & $\begin{array}{c}4.44^{b c} \pm \\
0.155\end{array}$ & $\begin{array}{c}4.5 \pm \\
0.0283\end{array}$ \\
\hline 10 & $\begin{array}{c}5.50^{b} \pm \\
0.103\end{array}$ & $\begin{array}{c}0.625^{\mathbf{b}} \pm \\
0.0101\end{array}$ & $\begin{array}{l}2.17^{c} \pm \\
0.0242\end{array}$ & $\begin{array}{l}1.84 * \pm \\
0.0135\end{array}$ & $\begin{array}{c}3.71^{e} \pm \\
6.91 \times 10^{-3}\end{array}$ & $\begin{array}{c}3.06 * \pm \\
0.026\end{array}$ & $\begin{array}{l}4.28 \mathrm{c} \pm \\
0.0846\end{array}$ & $\begin{array}{l}4.28 \pm \\
0.0388\end{array}$ \\
\hline
\end{tabular}

\footnotetext{
* Results given as (average \pm SE) x 1000 . Values in each individual column with the same letters are not significantly different (Tukey's Studentized Range (HSD) and Scheffe's Test $(\alpha \leq 0.05)$ ). Expected values with an asterisk are significantly different from observed values $(\alpha \leq 0.05)$.
} 
HPP of papaya nectar resulted in both significant increases and decreases in PME activity depending on the pressure-temperature combination examined (Figure 2). As pressure increased to $400 \mathrm{MPa}$ at $20^{\circ} \mathrm{C}$ and $60^{\circ} \mathrm{C}$, PME activity significantly increased $15.7 \%$ and $10.0 \%$, respectively. At 600 MPa PME activity significantly decreased $16.7 \%$ at $20^{\circ} \mathrm{C}$ and $11.6 \%$ at $60^{\circ} \mathrm{C}$. This significant decrease in PME activity, however, resulted in PME activities which were not significantly different from atmospheric controls. Enhancement of PME activity was also seen in research conducted by Fraeye et al. [49] wherein optimal recombinant Aspergillus aculeatus PME catalyzed pectin de-esterification was observed between $200-300 \mathrm{MPa}$ at $45-55^{\circ} \mathrm{C}$ and maximal tomato juice PME activity occurred at $300 \mathrm{MPa}$ at $50^{\circ} \mathrm{C}$ [50]. Gel formation was not prevented after any pressure-temperature combinations examined (Table 2). At both $20^{\circ} \mathrm{C}$ and $60^{\circ} \mathrm{C}$, gel formation was enhanced at $400 \mathrm{MPa}$ with strong gels forming within 48 hours, and slowed at $600 \mathrm{MPa}$ with gel formation prevented after 24 hours. Trends in gel formation followed closely with enhancement and inactivation of PME activity at 400 and $600 \mathrm{MPa}$, respectively.

Papaya PME activity was significantly impacted by irradiation (Table 5). As irradiation dose increased to 10 kGy, papaya PME activity experienced a significant 38.1\% reduction. However, reductions were not able to prevent gel formation in papaya nectar (Table 3), consistent with research carried out by D'Innocenzo and Lajolo [51] on papaya. Parker et al. [6] also reported that processing of papaya at $5 \mathrm{kGy}$ and $7.5 \mathrm{kGy}$ was unable to prevent gel formation. This indicates that irradiation on its own minimally impacts gel formation at doses examined.

PME activity was significantly reduced by all processing techniques, but traditional thermal processing was most effective, preventing gel formation at $110^{\circ} \mathrm{C}$ for 5 minutes; as temperature increased, rate of inactivation of PME activity increased. Similar reductions in PME activity were observed after UHT with processing at $110^{\circ} \mathrm{C}$ and $135^{\circ} \mathrm{C}$, effectively preventing gel formation in papaya nectar. HPP acted to significantly enhance PME activity at $400 \mathrm{MPa}$ and significantly inactivate PME at $600 \mathrm{MPa}$, but was unable to prevent gel formation in papaya nectar. Similarly, irradiation was unable to prevent gel formation in papaya nectar at all doses examined.

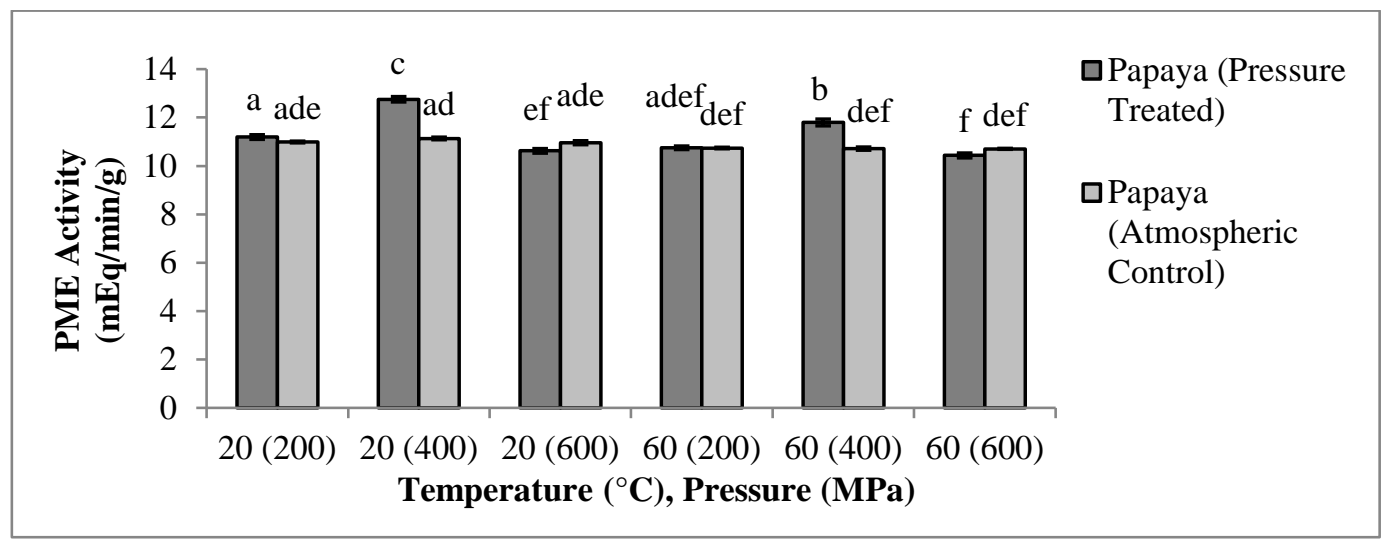

Figure 2. PME activity (mEqu $\mathrm{NaOH} / \mathrm{min} / \mathrm{g}$ ) for papaya nectar after high pressure processing (HPP). Results given as average \pm SE x 1000. Values with the same letters are not significantly different (Tukey's Studentized Range (HSD) $(\alpha \leq 0.05))$.

\subsection{Impact of processing on PME activity in papaya and strawberry nectar blends}

Significant PME activity reduction was seen in both $100 \%$ papaya and strawberry nectar controls and their respective blends after UHT processing (Table 5). PME activity in the 25P:75S blend was significantly reduced (44.9\%) at $80^{\circ} \mathrm{C}$ and encountered equivalent significant reductions of $93.6 \%$ at both $110^{\circ} \mathrm{C}$ and $135^{\circ} \mathrm{C}$. Similar PME reduction was observed in the $50 \mathrm{P}: 50 \mathrm{~S}$ blend, being significantly reduced $(45.0 \%)$ from $20^{\circ} \mathrm{C}$ to $80^{\circ} \mathrm{C}$, and encountered equivalent significant reductions of $96.0 \%$ at both $110^{\circ} \mathrm{C}$ and $135^{\circ} \mathrm{C}$. The $75 \mathrm{P}: 25 \mathrm{~S}$ blend, however, experienced a greater significant reduction (93.7\%) as temperature increased from $20^{\circ} \mathrm{C}$ to $80^{\circ} \mathrm{C}$, and nearly equivalent inactivations of $96.9 \%$ and $97.5 \%$ at $110^{\circ} \mathrm{C}$ and $135^{\circ} \mathrm{C}$, respectively. At $110^{\circ} \mathrm{C}$ and $135^{\circ} \mathrm{C}$, significant reductions of $98.0 \%$ and $98.8 \%$ occurred in the $100 \%$ papaya control, respectively. PME activity in the $100 \%$ strawberry control significantly decreased $(43.1 \%)$ from $20^{\circ} \mathrm{C}$ to $80^{\circ} \mathrm{C}$, and at $110^{\circ} \mathrm{C}$ and $135^{\circ} \mathrm{C}$ similar significant reductions of $88.5 \%$ and $93.0 \%$ were observed, respectively. As the percentage of papaya increased, reduction of PME at elevated temperatures also slightly increased. This corresponds with the trends observed in the $100 \%$ controls, wherein papaya PME activity reduction was less than strawberry at $80^{\circ} \mathrm{C}$, but as temperature increased, the degree to which PME activity was reduced in papaya, increased. Overall PME reduction in the blends was similar to the $100 \%$ controls at $110^{\circ} \mathrm{C}$ and $135^{\circ} \mathrm{C}$, but greater at $80^{\circ} \mathrm{C}$, especially in the $75 \mathrm{P}: 25 \mathrm{~S}$ blend, highlighting the benefit of blending together papaya and strawberry nectars upon UHT processing.

Across all blends, 25P:75S, 50P:50S, and 75P:25S, controls at $20^{\circ} \mathrm{C}$ experienced additive relationships between observed and expected values (Table 5). This means that blending of the fruit nectars without processing did not significantly affect PME activity. At $80^{\circ} \mathrm{C}$, however, all blends experienced synergistic reductions, with observed 
reductions greater than expected values. These synergistic reductions support the increased reduction in PME activity observed at $80^{\circ} \mathrm{C}$ compared to the $100 \%$ controls. In contrast, at $110^{\circ} \mathrm{C}$ and $135^{\circ} \mathrm{C}$, antagonistic enzyme reduction was experienced. These results may indicate that PME activity is slightly increased at elevated temperatures compared to expected values, but the observed antagonism is most likely a result of the detection sensitivity of the assay used to quantify PME activity.

Slight gelling occurred in the $100 \%$ papaya control but no gel formation was observed in the 25P:75S blend, suggesting that this ratio of papaya to strawberry nectar is effective at preventing gel formation without UHT (Table 4). At $20^{\circ} \mathrm{C}$, both the 50P:50S and 75P:25S blends experienced slight gelling after 72 hours, but after UHT processing at $80^{\circ} \mathrm{C}$, gel formation was prevented in both blends. This suggests that processing at $80^{\circ} \mathrm{C}$ is sufficient for reducing PME concentrations to low enough levels in the 50P:50S and 75P:25S blends to prevent gel formation, which is lower than $110^{\circ} \mathrm{C}$ required for the $100 \%$ papaya control.

PME activity in 100\% papaya, 100\% strawberry, and their respective blends was significantly reduced with increasing irradiation dose, but to slightly different degrees depending on the ratio of nectars examined (Table 5). In the 25P:75S blend PME activity was significantly reduced, 21.5\%, starting at $7.5 \mathrm{kGy}$. To a lesser degree, PME activity was also significantly reduced in the 50P:50S and 75P:25S blends with reductions of $13.3 \%$ starting at 2.5 $\mathrm{kGy}$, and $15.1 \%$ starting at $7.5 \mathrm{kGy}$, respectively. The $100 \%$ papaya control experienced the greatest reduction in PME activity, 38.1\%, starting at $2.5 \mathrm{kGy}$ while the 100\% strawberry control experienced the least reduction in PME activity, 12.4\%, starting at $5 \mathrm{kGy}$. Based on PME reduction alone, the ratio of nectars in the 25P:75S blend allowed for the greatest reduction in PME activity among all blends.

Upon closer examination, however, observed reductions in enzyme activity for the 25P:75S blend were statistically indifferent from expected reductions at $0 \mathrm{kGy}$, but at $2.5 \mathrm{kGy}, 5 \mathrm{kGy}, 7.5 \mathrm{kGy}$, and $10 \mathrm{kGy}$, observed reductions were significantly less than expected (Table 5), suggesting an antagonistic effect on inactivation. Similarly, at $2.5 \mathrm{kGy}, 5 \mathrm{kGy}, 7.5 \mathrm{kGy}$ and $10 \mathrm{kGy}$ observed reductions were also less than expected in the 50P:50S blend, but at $0 \mathrm{kGy}$ the observed activity was significantly less than expected. Unlike the other two blends, the 75P:25S blend had observed reductions greater than expected at $0 \mathrm{kGy}$, but at $2.5 \mathrm{kGy}, 5 \mathrm{kGy}, 7.5 \mathrm{kGy}$, and 10 kGy, observed reductions were indifferent from expected values. This means that even though the 75P:25S blend had slightly less overall PME activity reduction compared to the 25P:75S blend, since additive relationships were observed in the 75P:25S blend as irradiation dose increased (as opposed to antagonistic reductions which were observed in the 25P:75S and 50P:50S blends), the ratio of nectars in the 75P:25S blend was optimal for overall PME reduction, but still less than the $100 \%$ papaya control.

Similar to the $100 \%$ strawberry control, the 25P:75S blend experienced no gel formation at any treatment level examined (Table 4). The 50P:50S blend, however, had slight gelling starting at $24 \mathrm{~h}$ for $0 \mathrm{kGy}$ through $7.5 \mathrm{kGy}$, but at $10 \mathrm{kGy}$ no gel formation was observed. This suggests that $10 \mathrm{kGy}$ of irradiation was effective at reducing PME activity to low enough concentrations that gel formation did not occur. Gel formation was not prevented in the papaya control after irradiation (consistent with research carried out by D'Innocenzo and Lajolo [51] and Parker et al. [11] on papaya nectar), but was prevented after $10 \mathrm{kGy}$ in the 50P:50S blend, indicating that blending of fruit nectars is beneficial in preventing PME induced gel formation in combination with irradiation. In the 75P:25S blend, slight gelling began after 24 hours with a strong gel forming after 48 hours (Table 4 ). At 2.5 kGy slight gelling began after 24 hours, but strong gel formation was prevented until 72 hours. Slight gelling occurred after 24 hours for irradiation doses greater than $5 \mathrm{kGy}$, which indicates that irradiation did impact gel formation in the 75P:25S blend, but not to low enough concentrations to prevent gel formation.

Overall, blending of papaya and strawberry nectars mainly resulted in desirable synergistic reduction in enzymatic activity. $\mathrm{pH}$ change may have a crucial impact on the reduction in PME activity due to the blending of the fruit nectars. The optimal pH for PME activity is 6.9-7[33]. Because strawberry has a lower pH than papaya, the lowered $\mathrm{pH}$ due to blending of the nectars may be responsible for the desirable synergistic relationships observed (Table 3-4). Additionally, dilution of PME and substrates stemming from lowered concentrations of papaya nectar may also be responsible for synergistic reductions observed in nectar blends. UHT was the most effective at reducing PME activity, with synergistic reduction coupled with gel prevention making $80^{\circ} \mathrm{C}$ the optimal temperature among the levels examined. As papaya percentage increased, a greater reduction in PME activity was observed at higher temperatures. Even though irradiation did not produce the same PME reduction as seen with UHT, it is important to note that the 50P:50S blend processed at $10 \mathrm{kGy}$ was the only blend to allow irradiative prevention of gel formation.

\subsection{Impact of processing on POD activity in strawberry nectar}

Traditional thermal processing significantly impacted POD activity in strawberry nectar (Figure 3). Across all processing times, increasing temperature from $80^{\circ} \mathrm{C}$ to $130^{\circ} \mathrm{C}$ had minimal impact on POD activity; however, as processing time increased across all temperatures, significant reduction in POD activity occurred. In support of this trend, Tomás-Barberán et al. [52] strongly recommended "long treatment times to ensure POD inactivation, as well as to control POD activity during further storage.” At all temperatures $\left(80^{\circ} \mathrm{C}, 110^{\circ} \mathrm{C}\right.$, and $\left.130^{\circ} \mathrm{C}\right)$, POD 
activity in strawberry nectar significantly decreased at 2 minutes with reductions of $18.1 \%$, 33.9\%, and $24.2 \%$, respectively (Figure 3). As processing time increased beyond 2 minutes, significant reduction in POD activity continued through 10 minutes. A similar experiment carried out on strawberries by Terefe et al., [13] reported complete inactivation of POD after 5 minutes at $70^{\circ} \mathrm{C}$, highlighting the variability between different varieties of strawberries.

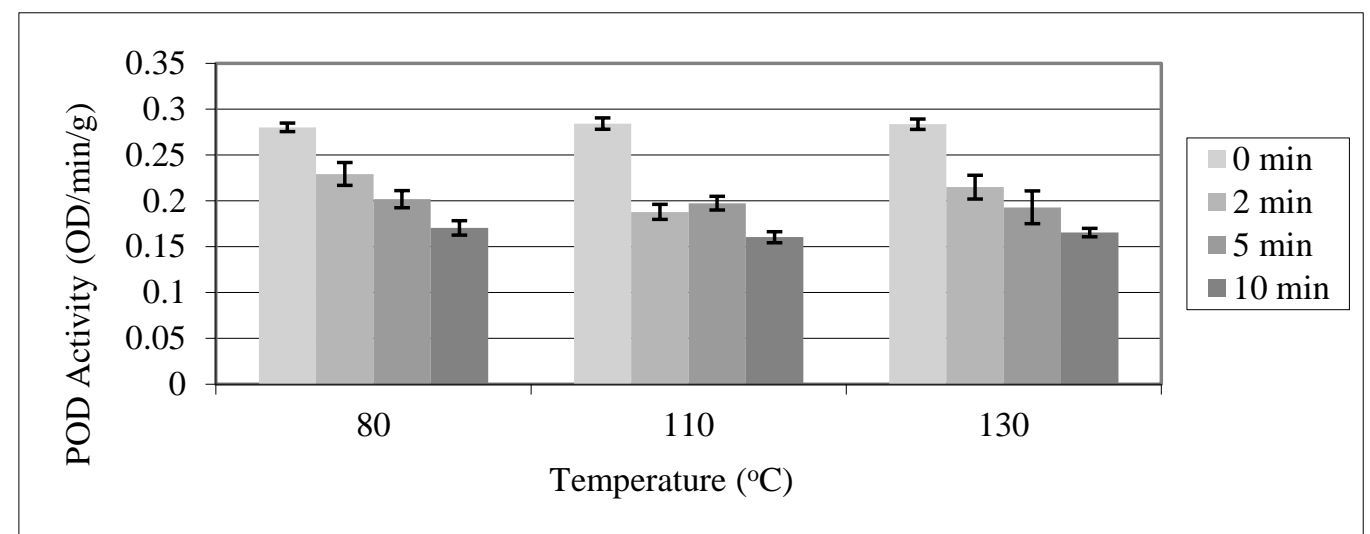

Figure 3. POD activity (Optical Density (OD)/min/g) for strawberry nectar after thermal processing. Results given as average \pm SE. Values with the same letters are not significantly different (Tukey’s Studentized Range (HSD) $(\alpha \leq 0.05))$.

Interestingly, UHT processing resulted in an overall increase in POD activity in strawberry nectar (Table 6). POD activity slightly increased from $20^{\circ} \mathrm{C}$ to $80^{\circ} \mathrm{C}$, significantly increased (13.2\%) at $110^{\circ} \mathrm{C}$, and then slightly decreased at $135^{\circ} \mathrm{C}$, with an overall slight $8.0 \%$ increase in POD activity. Even though POD activity was significantly enhanced at $110^{\circ} \mathrm{C}$ in strawberry, at $135^{\circ} \mathrm{C}$ activity was not significantly different from the control at $20^{\circ} \mathrm{C}$. In a study on POD activity in apricot nectar, there was also a significant increase in activity after UHT for $110^{\circ} \mathrm{C}$ for 8.6 seconds. Complete inactivation of POD activity in apricots occurred after processing 10 minutes at $85^{\circ} \mathrm{C}$ suggesting that extended processing time may be more effective at reducing POD activity compared to short processing times utilized in UHT [53].

When fruit nectar was subject to HPP, there was no significant difference between pressure treated samples and atmospheric controls, indicating that pressure alone has minimal impact on POD activity in strawberry nectar (Fig 4). As pressure increased and temperature remained constant at $20^{\circ} \mathrm{C}$, a significant reduction (15.6\%) in POD activity occurred at $600 \mathrm{MPa}$. Similarly, at $60^{\circ} \mathrm{C}$, a significant reduction (18.1\%) in POD activity occurred at 600 $\mathrm{MPa}$. At atmospheric pressure, an insignificant reduction $\left(7.60 \%\right.$ ) was observed at $20^{\circ} \mathrm{C}$, and at $60^{\circ} \mathrm{C}$ a significant reduction (15.6\%) in POD activity occurred. This highlights the fact that application of temperature alone resulted in loss of POD activity (11.8\%), significant at $60^{\circ} \mathrm{C}$, but in combination with pressure the reduction was greater (20.8\%). A study carried out by Garcia-Palazon et al. [54] found that strawberry POD activity increased by $13 \%$ and $1 \%$ at $400 \mathrm{MPa}$ for 5 and 10 minutes, respectively. This highlights the variability resulting from different fruit varietals at different pressure-temperature-time combinations. The greatest inactivation of POD occurred at $60^{\circ} \mathrm{C}-$ $600 \mathrm{MPa}$ (Figure 4), with similar substantial POD inactivation seen near this pressure in research conducted by Terefe et al. [13], and at $20^{\circ} \mathrm{C}, 600 \mathrm{MPa}$ pressure had the greatest effect on reducing POD activity, since no significant change was observed in the atmospheric control at the same temperature (Figure 4). Interestingly, the same reduction in POD activity was achieved with the pressure treated samples at $20^{\circ} \mathrm{C}$ as the atmosphere control at $60^{\circ} \mathrm{C}$. This suggests that ambient temperature coupled with pressure has similar effects as elevated temperatures at $60^{\circ} \mathrm{C}$ alone, but elevated temperatures combined with pressure results in the greatest inactivation of POD activity overall.

There was no significant change in POD activity in strawberry nectar after all irradiation doses examined (Table 6). Tomás-Barberan et al. [52] demonstrated that low doses of irradiation had mixed results on POD activity. 1.75$2.5 \mathrm{kGy}$ of irradiation caused increase in peach enzymatic activity [55]. In contrast, Wahid [56] reported no significant change in POD activity in mushrooms immediately after processing.

Overall, traditional thermal processing resulted in the greatest reduction in enzyme activity compared to all other processing techniques. Increasing temperature had minimal effect on POD activity, but as processing time increased, significant reductions in activity were observed across all temperatures. In contrast, UHT resulted in significantly enhanced POD activity making this form of processing undesirable for strawberry nectar. Both temperature and pressure impacted POD activity in strawberry nectar after HPP. Irradiation resulted in no significant change in POD activity compared to the control. 
Table 6. POD activity (Optical Density (OD)/min/g) for 100\% papaya and strawberry nectar controls and their respective blends (25P:75S, 50P:50S, 75P:25S) after ultra high temperature (UHT) processing and irradiation processing.

\begin{tabular}{|c|c|c|c|c|c|c|c|c|}
\hline & \multirow[t]{2}{*}{$100 \mathrm{P}$} & \multirow[t]{2}{*}{$100 \mathrm{~S}$} & \multicolumn{2}{|c|}{ 25P:75S } & \multicolumn{2}{|c|}{ 50P:50S } & \multicolumn{2}{|c|}{ 75P:25S } \\
\hline & & & Observed & Expected & Observed & Expected & Observed & Expected \\
\hline \multicolumn{9}{|c|}{$\begin{array}{l}\text { Temperature } \\
\left({ }^{\circ} \mathrm{C}\right)\end{array}$} \\
\hline 20 & $\begin{array}{c}0.714^{a} \pm \\
1.25 \times 10^{-2}\end{array}$ & $\begin{array}{c}0.494^{\mathrm{a}} \pm \\
9.17 \times 10^{-3}\end{array}$ & $\begin{array}{c}0.822^{a} \pm \\
1.00 \times 10^{-3}\end{array}$ & $\begin{array}{l}0.549 * \pm \\
8.04 \times 10^{-3}\end{array}$ & $\begin{array}{c}0.839^{a} \pm \\
7.72 \times 10^{-3}\end{array}$ & $\begin{array}{l}0.604 * \pm \\
9.51 \times 10^{-3}\end{array}$ & $\begin{array}{c}0.791^{a} \pm \\
7.68 \times 10^{-3}\end{array}$ & $\begin{array}{l}0.659 * \pm \\
1.27 \times 10^{-2}\end{array}$ \\
\hline 80 & $\begin{array}{c}0.630^{a} \pm \\
6.79 \times 10^{-3}\end{array}$ & $\begin{array}{l}0.550^{\mathrm{ab}} \pm \\
7.31 \times 10^{-3}\end{array}$ & $\begin{array}{c}0.754^{b} \pm \\
3.34 \times 10^{-4}\end{array}$ & $\begin{array}{l}0.570^{*} \pm \\
5.93 \times 10^{-3}\end{array}$ & $\begin{array}{l}0.801^{a b_{ \pm}} \\
5.00 \times 10^{-3}\end{array}$ & $\begin{array}{l}0.590 * \pm \\
5.82 \times 10^{-3}\end{array}$ & $\begin{array}{c}0.780^{a} \pm \\
1.46 \times 10^{-2}\end{array}$ & $\begin{array}{l}0.610^{*} \pm \\
7.03 \times 10^{-3}\end{array}$ \\
\hline 110 & $\begin{array}{c}0.589^{a} \pm \\
1.30 \times 10^{-2}\end{array}$ & $\begin{array}{c}0.569^{\mathrm{b}} \pm \\
4.36 \times 10^{-3}\end{array}$ & $\begin{array}{l}0.773^{a b} \pm \\
6.91 \times 10^{-3}\end{array}$ & $\begin{array}{l}0.574^{*} \pm \\
6.63 \times 10^{-3}\end{array}$ & $\begin{array}{l}0.763^{a b} \pm \\
9.72 \times 10^{-3}\end{array}$ & $\begin{array}{l}0.579 * \pm \\
9.28 \times 10^{-3}\end{array}$ & $\begin{array}{c}0.856^{a} \pm \\
7.95 \times 10^{-3}\end{array}$ & $\begin{array}{l}0.584 * \pm \\
1.31 \times 10^{-2}\end{array}$ \\
\hline 135 & $\begin{array}{c}0.639^{a} \pm \\
7.86 \times 10^{-3}\end{array}$ & $\begin{array}{l}0.537^{\mathrm{ab}} \pm \\
3.07 \times 10^{-3}\end{array}$ & $\begin{array}{c}0.702^{c} \pm \\
7.22 \times 10^{-3}\end{array}$ & $\begin{array}{l}0.562^{*} \pm \\
3.49 \times 10^{-3}\end{array}$ & $\begin{array}{c}0.752^{b} \pm \\
3.75 \times 10^{-3}\end{array}$ & $\begin{array}{l}0.588^{*} \pm \\
5.46 \times 10^{-3}\end{array}$ & $\begin{array}{c}0.711^{a} \pm \\
1.50 \times 10^{-2}\end{array}$ & $\begin{array}{c}0.613 \pm \\
7.90 \times 10^{-3}\end{array}$ \\
\hline \multicolumn{9}{|c|}{$\begin{array}{c}\text { Radiation } \\
\text { Dose (kGy) }\end{array}$} \\
\hline 0 & $\begin{array}{c}0.340^{a} \pm \\
8.13 \times 10^{-3}\end{array}$ & $\begin{array}{c}0.322^{\mathrm{a}} \pm \\
1.53 \times 10^{-2}\end{array}$ & $\begin{array}{c}0.347^{a} \pm 4.32 \\
\quad x 10^{-3}\end{array}$ & $\begin{array}{c}0.327 \pm \\
5.81 \times 10^{-3}\end{array}$ & $\begin{array}{c}0.372^{a} \pm 2.20 \\
\times 10^{-2}\end{array}$ & $\begin{array}{c}0.331 \pm \\
4.32 \times 10^{-3}\end{array}$ & $\begin{array}{c}0.411^{a} \pm 8.91 \mathrm{x} \\
10^{-3}\end{array}$ & $\begin{array}{l}0.336^{*} \pm \\
3.60 \times 10^{-3}\end{array}$ \\
\hline 2.5 & $\begin{array}{c}0.356^{a b} \pm \\
8.73 \times 10^{-3}\end{array}$ & $\begin{array}{c}0.300^{\mathrm{a}} \pm \\
1.26 \times 10^{-2}\end{array}$ & $\begin{array}{c}0.308^{b} \pm 9.06 \\
\times 10^{-3}\end{array}$ & $\begin{array}{c}0.314 \pm \\
4.85 \times 10^{-3}\end{array}$ & $\begin{array}{c}0.350^{a b} \pm 1.97 \\
\times 10^{-2}\end{array}$ & $\begin{array}{c}0.328 \pm \\
3.84 \times 10^{-3}\end{array}$ & $\begin{array}{c}0.375^{a} \pm 6.22 \times \\
10^{-3}\end{array}$ & $\begin{array}{l}0.342^{*} \pm \\
3.63 \times 10^{-3}\end{array}$ \\
\hline 5 & $\begin{array}{c}0.381^{a b} \pm \\
1.72 \times 10^{-2}\end{array}$ & $\begin{array}{c}0.306^{\mathrm{a}} \pm \\
4.36 \times 10^{-3}\end{array}$ & $\begin{array}{c}0.309^{b} \pm 5.96 \\
\times 10^{-3}\end{array}$ & $\begin{array}{l}0.325^{*} \pm \\
2.70 \times 10^{-3}\end{array}$ & $\begin{array}{c}0.276^{b c} \pm \\
1.84 \times 10^{-2}\end{array}$ & $\begin{array}{l}0.343^{*} \pm \\
4.43 \times 10^{-3}\end{array}$ & $\begin{array}{c}0.332^{a} \pm 2.14 \mathrm{x} \\
10^{-2}\end{array}$ & $\begin{array}{c}0.362 \pm \\
6.46 \times 10^{-3}\end{array}$ \\
\hline 7.5 & $\begin{array}{c}0.407^{b} \pm \\
1.42 \times 10^{-2}\end{array}$ & $\begin{array}{c}0.323^{\mathrm{a}} \pm \\
1.07 \times 10^{-2}\end{array}$ & $\begin{array}{c}0.319^{a b} \pm 1.03 \\
\times 10^{-2}\end{array}$ & $\begin{array}{l}0.344^{*} \pm \\
4.39 \times 10^{-3}\end{array}$ & $\begin{array}{c}0.268^{c} \pm 4.51 \\
\times 10^{-3}\end{array}$ & $\begin{array}{l}0.365^{*} \pm \\
4.44 \times 10^{-3}\end{array}$ & $\begin{array}{c}0.353^{a} \pm 3.23 x \\
10^{-2}\end{array}$ & $\begin{array}{c}0.386 \pm \\
5.49 \times 10^{-3}\end{array}$ \\
\hline 10 & $\begin{array}{c}0.327^{a} \pm \\
1.39 \times 10^{-2}\end{array}$ & $\begin{array}{c}0.318^{\mathrm{a}} \pm \\
5.74 \times 10^{-3}\end{array}$ & $\begin{array}{c}0.351^{a} \pm 8.91 \\
\mathrm{x} 10^{-3}\end{array}$ & $\begin{array}{l}0.320 * \pm \\
2.76 \times 10^{-3}\end{array}$ & $\begin{array}{c}0.394^{a} \pm 7.95 \\
\times 10^{-3}\end{array}$ & $\begin{array}{l}0.322 * \pm \\
3.76 \times 10^{-3}\end{array}$ & $\begin{array}{c}0.398^{a} \pm 5.78 \\
\times 10^{-3}\end{array}$ & $\begin{array}{l}0.325^{*} \pm \\
5.26 \times 10^{-3}\end{array}$ \\
\hline
\end{tabular}

* Results given as average \pm SE. Values in each individual column with the same letters are not significantly different (Tukey's Studentized Range (HSD) $(\alpha \leq 0.05))$. Expected values with an asterisk are significantly different from observed values $(\alpha \leq$ $0.05)$.

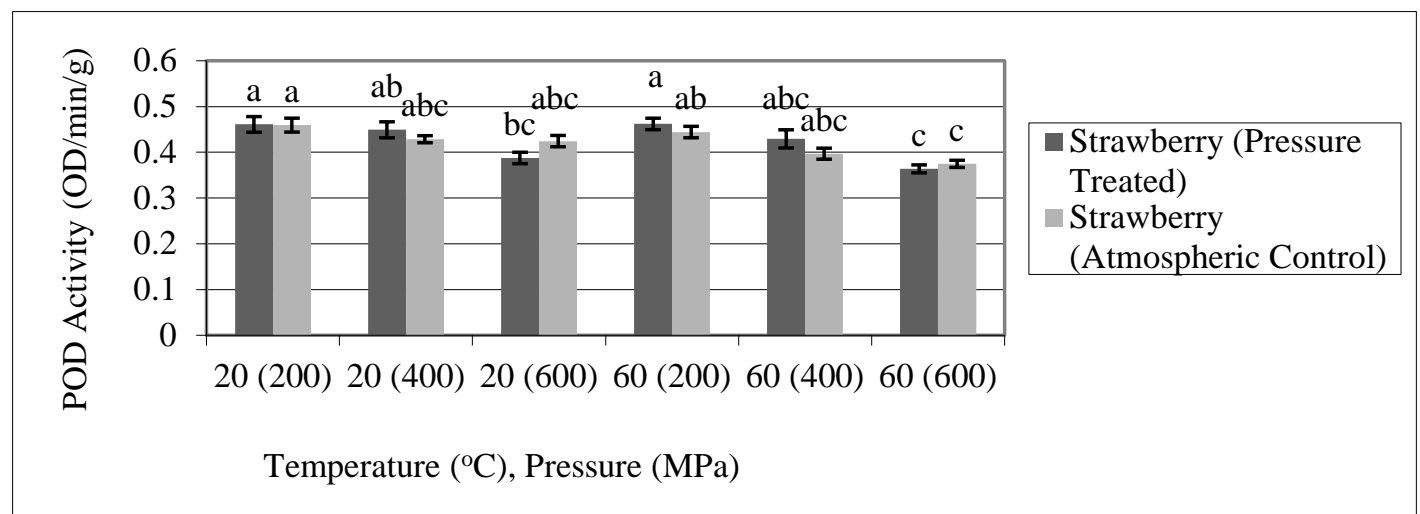

Figure 4. POD activity (Optical Density (OD)/min/g) for strawberry nectar after high pressure processing (HPP). Results given as average \pm SE. Values with the same letters are not significantly different (Tukey's Studentized Range (HSD) $(\alpha \leq 0.05))$.

\subsection{Impact of processing on POD activity in papaya and strawberry nectar blends}

The effect of UHT on POD activity was dependent on the ratio of nectars examined (Table 6). In the case of the 25P:75S blend, POD activity significantly decreased from $20^{\circ} \mathrm{C}$ to $80^{\circ} \mathrm{C}$, remained relatively constant at $110^{\circ} \mathrm{C}$, and significantly decreased at $135^{\circ} \mathrm{C}$, with an overall $14.5 \%$ reduction in POD activity. Similarly, POD activity in the 50P:50S blend significantly decreased starting at $135^{\circ} \mathrm{C}$, with an overall $10.3 \%$ reduction. Unlike the other two blends, POD activity in the $75 \mathrm{P}: 25 \mathrm{~S}$ blend slightly decreased from $20^{\circ} \mathrm{C}$ to $80^{\circ} \mathrm{C}$, slightly increased at $110^{\circ} \mathrm{C}$, and then slightly decreased again at $135^{\circ} \mathrm{C}$. Even though a $10.2 \%$ decrease in POD activity was observed overall, trends observed for the 75P:25S blend were insignificant. As the ratio of papaya increased, reduction in POD activity also decreased, more closely mirroring the $100 \%$ papaya control. Since all blends resulted in an overall reduction 
in POD activity, significant at times, this suggests that blending of papaya and strawberry nectars beneficially reduces POD activity to a greater extent than $100 \%$ strawberry alone, and similarly to $100 \%$ papaya alone.

Antagonistic reductions were present in all blends at nearly every treatment level (Table 6). Observed POD activity reduction was significantly less than expected across all temperatures in both the 25P:75S blend and 50P:50S blend. Similarly, observed POD activity reduction in the 75P:25S blend was significantly less than expected at $20^{\circ} \mathrm{C}, 80^{\circ} \mathrm{C}$, and $110^{\circ} \mathrm{C}$, while observed POD activity reduction was significantly indifferent at $135^{\circ} \mathrm{C}$.

The ratio of papaya to strawberry in nectar blends impacts the extent of POD activity after irradiation (Table 6) .Unlike the $100 \%$ controls, as irradiation dose increased, POD activity in the 25P:75S blend significantly decreased at $2.5 \mathrm{kGy}$ and $5 \mathrm{kGy}(11.3 \%)$, then significantly increased at $10 \mathrm{kGy}$, resulting in an overall slight increase of $1.04 \%$. A similar overall increase in POD activity was observed in the 50P:50S blend, slightly decreasing from $0 \mathrm{kGy}$ to $2.5 \mathrm{kGy}$, significantly decreasing at $5 \mathrm{kGy}$ and $7.5 \mathrm{kGy}(28.0 \%)$ and then significantly increasing with an overall slight net increase of 3.63\% in POD activity. Similar to the $100 \%$ strawberry control, irradiation was unable to significantly reduce POD activity in the 75P:25S blend. Reduction in POD activity, significant at times, tends to occur between $2.5 \mathrm{kGy}$ and $7.5 \mathrm{kGy}$, but with increasing irradiation dose an increase in POD activity occurs to a greater extent than either of the $100 \%$ controls, with reasons for this further explained by pulp structure breakdown allowing previously bound substrate for POD to be more readily available due to the softening fruit matrix [57].

Observed reductions for the 25P:75S blend at $0 \mathrm{kGy}$ and $2.5 \mathrm{kGy}$ were not significantly different from expected reductions; at $5 \mathrm{kGy}$ and $7.5 \mathrm{kGy}$ observed reductions were significantly greater than expected, and at $10 \mathrm{kGy}$ the observed reduction was significantly less than expected (Table 6). A similar trend was seen with the 50P:50S blend wherein observed reductions were not significantly different from expected reductions at $0 \mathrm{kGy}$ and $2.5 \mathrm{kGy}$, at 5 kGy and 7.5 kGy observed reductions were significantly greater than expected, and at $10 \mathrm{kGy}$ the observed reduction was significantly less than expected. Unlike the other blends, the 75P:25S blend had observed reductions significantly less than expected at $0 \mathrm{kGy}, 2.5 \mathrm{kGy}$ and $10 \mathrm{kGy}$ and at $5 \mathrm{kGy}$ and $7.5 \mathrm{kGy}$ there was no significant difference between observed and expected reductions. The increase in POD activity at $10 \mathrm{kGy}$, discussed above, is supported by the fact that antagonistic reduction was experienced at $10 \mathrm{kGy}$ in all blends. These findings also highlight that blending together papaya and strawberry, specifically the 25P:75S and 50P:50S blends, results in greater reduction in POD activity than the $100 \%$ controls at $5 \mathrm{kGy}$ and $7.5 \mathrm{kGy}$ of irradiation where synergistic reductions were observed. Regardless of ratio of papaya to strawberry, processing between $2.5 \mathrm{kGy}$ and $7.5 \mathrm{kGy}$ generally acts to best reduce POD activity in the blends with exposure to $10 \mathrm{kGy}$ counteracting this loss due to increase in POD activity [57].

Overall, UHT processing of the nectar blends mainly resulted in antagonistic reductions in POD activity. However, this antagonism was mainly attributed to interactions between the fruit blends, not UHT. Unlike UHT, irradiation was more effective at reducing POD activity in the nectar blends. Synergistic reductions observed at 5 kGy and $7.5 \mathrm{kGy}$, specifically in the 25P:75S and 50P:50S blends, resulted in the greatest POD activity reduction, greater than either fruit processed on their own.

\section{Conclusions}

Traditional thermal and UHT processing were able to sufficiently reduce PME activity to completely prevent gel formation in papaya nectar when processed at $110^{\circ} \mathrm{C}$ for 5 minutes and $110^{\circ} \mathrm{C}$ for $1-3$ seconds, respectively. PME reduction in nectar blends was enhanced by synergistic reductions after UHT at $80^{\circ} \mathrm{C}$. HPP of papaya nectar was able to enhance PME activity at $400 \mathrm{MPa}$, and significantly decrease activity at $600 \mathrm{MPa}$, but was unable to prevent gel formation. The 25P:75S blend was able to prevent gel formation without processing due to dilution of the papaya nectar. Irradiation was unable to prevent gel formation in 100\% papaya nectar. The 50P:50S blend processed at $10 \mathrm{kGy}$, however, was the only blend to allow irradiative prevention of gel formation. UHT was the only technique resulting in an increase in POD activity. This increase, coupled with antagonistic reductions makes UHT undesirable for POD inactivation in strawberry nectar. HPP of strawberry nectar at elevated temperatures and pressures was successful in reducing POD activity, with increased pressure slightly enhancing the effectiveness of HPP compared to the atmospheric control. Irradiation was ineffective at reducing strawberry POD activity. Synergistic reductions of POD activity in nectar blends after irradiation resulted in enhanced reduction in POD activity to an extent, but any significant reductions were negated at higher irradiation doses. The reason for this can be attributed to the softening of the fruit matrix, known as pulp structure breakdown, releasing substrate, which can readily be utilized by POD [57]. Processing strawberry 2 minutes at all temperatures examined resulted in slightly greater reductions in POD activity than HPP and irradiation.

For both PME and POD, traditional thermal processing was most effective at inactivation, with UHT sufficiently reducing PME activity in papaya nectar and both HPP and irradiation reducing POD activity in strawberry nectar, similar to traditional thermal processing at low temperatures. Blending of papaya and strawberry nectars mainly resulted in desirable synergistic reduction in enzymatic activity. 


\section{Acknowledgements}

Jeffrey Swada was supported by a College of Agriculture, Consumer, and Environmental Sciences Jonathan Baldwin Turner Graduate Fellowship.

\section{References}

[1] Wu X, Beecher GR, Holden JM, Haytowitz DB, Gebhardt SE, Prior RL. Lipophilic and hydrophilic antioxidant capacities of common foods in the United States. Journal of Agricultural and Food Chemistry. 2004;52(12):4026-4037.

[2] Joseph JA, Shukitt-Hale B, Willis LM. Grape juice, berries, and walnuts affect brain aging and behavior. The Journal of Nutrition. 2009;139(9):1813S-1817S.

[3] Corral RD, Yahia EM, Carrillo A, González G. The correlation between some nutritional components with total antioxidant capacity (measured with six different assays) in eight horticultural commodities. In: VI International Postharvest Symposium (Acta Horticulture 877). 2010. p. 1267-1274.

[4] Moraga G, Martínez-Navarrete N, Chiralt A. Compositional changes of strawberry due to dehydration, cold storage and freezing-thawing processes. Journal of Food Processing and Preservation. 2006;30(4):458-474.

[5] Fraeye I, Knockaert G, Van Buggenhout S, Duvetter T, Hendrickx M, Van Loey A. Enzyme infusion and thermal processing of strawberries: Pectin conversions related to firmness evolution. Food Chemistry. 2009;114(4):1371-1379.

[6] Parker TL, Esgro ST, Miller SA, Myers LE, Meister RA, Toshkov SA, Engeseth NJ. Development of an optimised papaya pulp nectar using a combination of irradiation and mild heat. Food Chemistry. 2010;118(3):861-869.

[7] Pelloux J, Rusterucci C, Mellerowicz EJ. New insights into pectin methylesterase structure and function. Trends in Plant Science. 2007;12(6):267-277.

[8] Lacroix N, Fliss I, Makhlouf J. Inactivation of pectin methylesterase and stabilization of opalescence in orange juice by dynamic high pressure. Food Research International. 2005;38(5):569-576.

[9] Prasanna V, Prabha TN, Tharanathan RN. Fruit ripening phenomena-an overview. Critical Reviews in Food Science and Nutrition. 2007;47(1):1-19.

[10] Slavov A, Garnier C, Crépeau MJ, Durand S, Thibault JF, Bonnin E. Gelation of high methoxy pectin in the presence of pectin methylesterases and calcium. Carbohydrate Polymers. 2009;77(4):876-884.

[11] Burnette FS. Peroxidase and its relationship to food flavor and quality: a review. Journal of Food Science. 1977;42(1):1-6.

[12] Cano MP, Hernandez A, De Ancos B. High pressure and temperature effects on enzyme inactivation in strawberry and orange products. Journal of Food Science. 1997;62(1):85-88.

[13] Terefe NS, Yang YH, Knoerzer K, Buckow R, Versteeg C. High pressure and thermal inactivation kinetics of polyphenol oxidase and peroxidase in strawberry puree. Innovative Food Science \& Emerging Technologies. 2010;11(1):52-60.

[14] Grambow HJ, Langenbeck-Schwich B. The relationship between oxidase activity, peroxidase activity, hydrogen peroxide, and phenolic compounds in the degradation of indole-3-acetic acid in vitro. Planta. 1983;157(2):132-137.

[15] Cheng GW, Breen PJ. Activity of phenylalanine ammonia-lyase (PAL) and concentrations of anthocyanins and phenolics in developing strawberry fruit. Journal of the American Society for Horticultural Science. 1991;116(5):865-869.

[16] Robinson DS. Peroxidases and catalases in foods. In: Robinson DS, Eskin NA (Eds.). Oxidative Enzymes in Foods. Amsterdam: Elsevier Applied Science;1991. p. 1-49.

[17] Häkkinen SH, Törrönen AR. Content of flavonols and selected phenolic acids in strawberries and Vaccinium species: influence of cultivar, cultivation site and technique. Food Research International. 2000;33(6):517524.

[18] Chisari M, Barbagallo RN, Spagna G. Characterization of polyphenol oxidase and peroxidase and influence on browning of cold stored strawberry fruit. Journal of Agricultural and Food Chemistry. 2007;55(9):34693476.

[19] Ahmed J, Shivhare US, Sandhu KS. Thermal degradation kinetics of carotenoids and visual color of papaya puree. Journal of Food Science. 2002;67(7):2692-2695.

[20] Anthon GE, Barrett DM. Kinetic parameters for the thermal inactivation of quality-related enzymes in carrots and potatoes. Journal of Agricultural and Food Chemistry. 2002;50(14):4119-4125.

[21] Tansakul A, Kantrong H, Saengrayup R, Sura P. Thermophysical properties of papaya puree. International Journal of Food Properties. 2012;15(5):1086-1100. 
[22] Verlent I, Smout C, Duvetter TM, Hendrickx ME, Van Loey A. Effect of temperature and pressure on the activity of purified tomato polygalacturonase in the presence of pectins with different patterns of methyl esterification. Innovative Food Science \& Emerging Technologies. 2005;6(3):293-303.

[23] Sanchez-Vega R, Mujica-Paz H, Marquez-Melendez R, Ngadi MO, Ortega-Rivas E. Enzyme inactivation on apple juice treated by ultrapasteurization and pulsed electric fields technology. Journal of Food Processing and Preservation. 2009;33(4):486-499.

[24] Falguera V, Pagán J, Ibarz A. Effect of UV irradiation on enzymatic activities and physicochemical properties of apple juices from different varieties. LWT-Food Science and Technology. 2011;44(1):115-119.

[25] Slausan DO, Cooper BJ. Mechanisms of disease: a textbook of comparative general pathology. (2nd ed.). Baltimore: Williams \& Wilkins;1990.

[26] Palozza P, Krinsky NI. $\beta$-Carotene and $\alpha$-tocopherol are synergistic antioxidants. Archives of Biochemistry and Biophysics. 1992;297(1):184-187.

[27] Fuhrman B, Volkova N, Rosenblat M, Aviram M. Lycopene synergistically inhibits LDL oxidation in combination with vitamin E, glabridin, rosmarinic acid, carnosic acid, or garlic. Antioxidants and Redox Signaling. 2000;2(3):491-506.

[28] Bates RP, Morris JR, Crandall PG. Principles and practices of small-and medium-scale fruit juice processing. Food \& Agriculture Org.; 2001.

[29] Pedrielli P, Skibsted LH. Antioxidant synergy and regeneration effect of quercetin,(-)-epicatechin, and (+)catechin on $\alpha$-tocopherol in homogeneous solutions of peroxidating methyl linoleate. Journal of Agricultural and Food Chemistry. 2002;50(24):7138-7144.

[30] Meletis CD, Barker JE. Synergy in nutrients. Alternative \& Complementary Therapies. 2004;10(6):326-329.

[31] Cirico TL, Omaye ST. Additive or synergetic effects of phenolic compounds on human low density lipoprotein oxidation. Food and Chemical Toxicology. 2006;44(4):510-516.

[32] Hazewindus M, Haenen GR, Weseler AR, Bast A. The anti-inflammatory effect of lycopene complements the antioxidant action of ascorbic acid and $\alpha$-tocopherol. Food Chemistry. 2012;132(2):954-958.

[33] Ly-Nguyen B, Van Loey AM, Fachin D, Verlent I, Duvetter T, Vu ST, Smout C, Hendrickx ME. Strawberry pectin methylesterase (PME): purification, characterization, thermal and high-pressure inactivation. Biotechnology Progress. 2002;18(6):1447-1450.

[34] FDA. Approximate $\mathrm{pH}$ of foods and food products, 2007. http://www.foodscience.caes.uga.edu/extension/ documents/fdaapproximatephoffoodslacf-phs.pdf. (Accessed Aug. 2014).

[35] Bahorun T, Luximon-Ramma A, Crozier A, Aruoma OI. Total phenol, flavonoid, proanthocyanidin and vitamin $\mathrm{C}$ levels and antioxidant activities of Mauritian vegetables. Journal of the Science of Food and Agriculture. 2004;84(12):1553-1561.

[36] Sila DN, Smout C, Vu TS, Hendrickx ME. Effects of high-pressure pretreatment and calcium soaking on the texture degradation kinetics of carrots during thermal processing. Journal of Food Science. 2004;69(5):E205211.

[37] Chang SY, Toledo RT. Simultaneous determination of thermal diffusivity and heat transfer coefficient during sterilization of carrot dices in a packed bed. Journal of Food Science. 1990;55(1):199-205.

[38] Fang Z, Zhang M, Tao G, Sun Y, Sun J. Chemical composition of clarified bayberry (Myrica rubra Sieb. et Zucc.) juice sediment. Journal of Agricultural and Food Chemistry. 2006;54(20):7710-7716.

[39] Sharoba AM, Bahlol HE, El-Desouky AI. Establishing a schedule to determine the optimal thermal process time for some canned fruit products. Annals of Agric. Sc. 2007;45(1):125-145.

[40] Reyes De Corcuera JI. (Personal communication). Department of Food Science and Technology, University of Georgia.

[41] US General Accounting Office. Food irradiation report: Available research indicates that benefits outweigh risks: report to congressional requesters. GAO/RCED-00-217.Washington, D.C.: USGAO. 2000.

[42] Follett PA, Armstrong JW. Revised irradiation doses to control melon fly, Mediterranean fruit fly, and oriental fruit fly (Diptera: Tephritidae) and a generic dose for tephritid fruit flies. Journal of Economic Entomology. 2004;97(4):1254-1262.

[43] World Health Organization. High-dose irradiation: wholesomeness of food irradiatied with doses above 10 kGy. World Health Organization; 1999.

[44] Thayer DW. Food irradiation: benefits and concerns. Journal of Food Quality. 1990;13(3):147-169.

[45] Chang LW, Morita LL, Yamamoto HY. Papaya pectinesterase inhibition by sucrose. Journal of Food Science. 1965;30(2):218-222.

[46] Liu X. (Personal communication). Department of Statistics, University of Illinois Urbana-Champaign.

[47] Sampedro F, Rodrigo D, Hendrickx M. Inactivation kinetics of pectin methyl esterase under combined thermal-high pressure treatment in an orange juice-milk beverage. Journal of Food Engineering. 2008;86(1):133-139. 
[48] Sampedro F, Geveke DJ, Fan X, Zhang HQ. Effect of PEF, HHP and thermal treatment on PME inactivation and volatile compounds concentration of an orange juice-milk based beverage. Innovative Food Science \& Emerging Technologies. 2009;10(4):463-469.

[49] Fraeye I, Duvetter T, Verlent I, Sila DN, Hendrickx M, Van Loey A. Comparison of enzymatic deesterification of strawberry and apple pectin at elevated pressure by fungal pectinmethylesterase. Innovative Food Science \& Emerging Technologies. 2007;8(1):93-101.

[50] Hsu KC. Evaluation of processing qualities of tomato juice induced by thermal and pressure processing. LWT-Food Science and Technology. 2008;41(3):450-459.

[51] D'innocenzo MA, Lajolo FM. Effect of gamma irradiation on softening changes and enzyme activities during ripening of papaya fruit. Journal of Food Biochemistry. 2001;25(5):425-438.

[52] Tomás-Barberán FA, Espín JC. Phenolic compounds and related enzymes as determinants of quality in fruits and vegetables. Journal of the Science of Food and Agriculture. 2001;81(9):853-876.

[53] Huang W, Bi X, Zhang X, Liao X, Hu X, Wu J. Comparative study of enzymes, phenolics, carotenoids and color of apricot nectars treated by high hydrostatic pressure and high temperature short time. Innovative Food Science \& Emerging Technologies. 2013;18:74-82.

[54] Garcia-Palazon A, Suthanthangjai W, Kajda P, Zabetakis I. The effects of high hydrostatic pressure on $\beta$ glucosidase, peroxidase and polyphenoloxidase in red raspberry (Rubus idaeus) and strawberry (Fragaria× ananassa). Food Chemistry. 2004;88(1):7-10.

[55] Shi JX, Zhang RY, Li XJ, Wang YX. The influence of post-harvest gamma irradiation on the activity of enzymes in peach fruit. Journal of Southwestern Agricultural University. 1993;5:157-161.

[56] Wahid M. Effect of gamma irradiation and storage on the catalase and peroxidase activities of mushrooms. Lebensmittel Wissenschaft \& Technologie.1980;13:291-292.

[57] Swada JG, Keeley CJ, Ghane MA, Engeseth NJ. Relationship between pulp structure breakdown and nutritional value of papaya (Carica papaya) and strawberry (Fragaria $\mathrm{x}$ ananassa) nectars using alternative thermal and non-thermal processing techniques. Journal of the Science of Food and Agriculture. 2016;96(7):2514-2523.

(C) 2020 by the author(s). This work is licensed under a Creative Commons Attribution 4.0 International License (http://creativecommons.org/licenses/by/4.0/). Authors retain copyright of their work, with first publication rights granted to Tech Reviews Ltd. 